研究

\title{
Effects of Hot Isostatic Pressing on Polarization Fatigue Characteristics of Lead-Based Ferroelectric Thin Films Crystallized from the Amorphous State
}

\author{
Masafumi Kobune, Shinichi Kojima, Yusuke Nishioka and Tetsuo Yazawa \\ Department of Material Science Engineering, Graduate School of Engineering, University of Hyogo, 2167 Shosha, Himeji 671-2201
}

Received November 11, 2003

\begin{abstract}
SYNOPSIS
Ferroelectric properties and memory characteristics of $\mathrm{Pt} / 0.24 \mathrm{~Pb}\left(\mathrm{Zn}_{1 / 3} \mathrm{Nb}_{2 / 3}\right) \mathrm{O}_{3} \cdot 0.384 \mathrm{PbZrO}_{3} \cdot 0.376 \mathrm{PbTiO}_{3}$ (PZNZT) $/ \mathrm{PbTiO}_{3} / \mathrm{Pt}$ capacitors fabricated by crystallizing amorphous $\mathrm{PZNZT}$ films at $500^{\circ} \mathrm{C}$ for $1 \mathrm{~h}$ under high pressures of 1.0-176.5 MPa using hot isostatic pressing (HIP) are investigated. The relative permittivity decreased linearly with increasing HIP pressure. All the samples treated at pressures greater than 17.7 MPa completely showed fatigue-free characteristics even after up to $3 \times 10^{10}$ cycles. From dielectric measurements and cross-sectional highresolution TEM images, these fatigue-free behaviors may be caused by that several elongated amorphous areas oriented parallel to the substrate formed near the interface between the crystallized PZNZT layer and the $\mathrm{PbTiO}_{3}$ (PT) seed layer, which would play an important role in restraining the diffusion of lead from the crystallized layer toward the PT seed layer.
\end{abstract}

KEYWORDS

PZN-PZ-PT, hot isostatic pressing, high-resolution transmission electron microscope, FeRAMs, polarization fatigue characteristic

\section{Introduction}

In recent years, high-pressure crystallization processes have attracted much attention as a promising technology for the crystallization of both lead-based and lead-free films in an amorphous state. It is well known that leakage current characteristics and surface flatness can be substantially improved through the use of such techniques in comparison to films crystallized by conventional rapid thermal annealing (RTA). Kakimi et al. ${ }^{1,2)}$ previously revealed that heat-treatment of $\mathrm{Bi}_{4} \mathrm{Ti}_{3} \mathrm{O}_{12}$ (BIT) films fabricated by spincoating pyrolysis under oxygen gas pressure of $4.0 \times 10^{5} \mathrm{~Pa}$ produces films with high $c$-axis orientation, as well as good surface morphology and ferroelectric properties. Kijima et al. ${ }^{3-5)}$ reported that the annealing of BIT films containing $\mathrm{Bi}_{2} \mathrm{SiO}_{5}$ (BSO) using a sol-gel spin-coating method under oxygen gas pressure of around 1.0 MPa produces a stable perovskite structure, resulting in excellent leakage current characteristics. The present authors have also investigated the physical properties of films crystallized under high oxygen pressures of $>1.0 \mathrm{MPa}$, and previously reported that hot isostatic pressing (HIP) of amorphous $0.24 \mathrm{~Pb}\left(\mathrm{Zn}_{1 / 3} \mathrm{Nb}_{2 \beta}\right) \mathrm{O}_{3}$. $0.384 \mathrm{PbZrO}_{3} \cdot 0.376 \mathrm{PbTiO}_{3}(\mathrm{PZNZT} 24 / 38.4 / 37.6)$ films in $\mathrm{Ar} / \mathrm{O}_{2}$ mixed gas at pressures of $1.8-176.5 \mathrm{MPa}$ affords films with almost uniform $c$-axis orientation and particularly excellent electric insulation in high electric fields of $400-$ $600 \mathrm{kV} / \mathrm{cm}^{\text {}}$. However, there have as yet been no reports on the dielectric, ferroelectric, and memory characteristics of PZNZT films that have been HIP-treated at relatively low temperature under a wide range of pressure conditions. PZNZT 24/38.4/37.6 ceramics fabricated by a conventional sintering process and a hybrid sintering process were shown in a previous report to exhibit excellent ferroelectric properties ${ }^{7,8}$. If it is indeed possible to fabricate thin PZNZT films by a high-pressure crystallization process as described above, the proposed materials can be expected to be highly useful in practical applications as ferroelectric random access memory (FeRAM).

In the present study, PZNZT 24/38.4/37.6 films with highly uniform $c$-axis orientation are fabricated on $\mathrm{PbTiO}_{3}$ (PT)/Pt(111)/SiO $/ 2 / \mathrm{Si}(100)$ substrates by the HIP crystallization process from the amorphous state. The dielectric, ferroelectric and polarization fatigue characteristics of the fabricated Pt/PZNZT/PT/Pt capacitors are also discussed.

\section{Experimental}

Amorphous PZNZT films were deposited on PT $(30 \mathrm{~nm}) /$ $\mathrm{Pt}(200 \mathrm{~nm}) / \mathrm{SiO}_{2}(400 \mathrm{~nm}) / \mathrm{Si}$ substrates by $\mathrm{rf}$ magnetron sputtering using a ceramic target and without heating the 
substrates. The sputtering conditions for fabrication of the amorphous PZNZT (a-PZNZT) films, the PT seed layer, and the top and bottom Pt electrodes were summarized in the previous report ${ }^{6}$. The PT seed layer was deposited on $\mathrm{Pt}(111) / \mathrm{SiO}_{2} / \mathrm{Si}(100)$ substrates by if magnetron sputtering at $570^{\circ} \mathrm{C}$ using a $\left(0.25 \mathrm{PbTiO}_{3}+0.75 \mathrm{PbO}\right)$ powder target. This seed layer had an almost uniform (100) orientation. As-deposited amorphous films were chemically oxidized by ultrasonication in $2.2 \mathrm{M}$ hydrogen-peroxide solution, followed by capsule-free $\mathrm{HIP}$ at $500^{\circ} \mathrm{C}$ for $1 \mathrm{~h}$ under $\mathrm{Ar} / \mathrm{O}_{2}$ at gas pressure of 1.0-176.5 MPa. The thickness of the PZNZT films after crystallization was around $270 \mathrm{~nm}$. The crystalline phase and the orientation of crystallized PZNZT films were investigated by X-ray diffractometry (XRD; RINT1500, Rigaku). The degree of $c$-axis orientation $(\alpha)$ for samples with tetragonal structure was calculated as described elsewhere). Energy-dispersive $\mathrm{X}$-ray fluorescence (XRF) analysis was employed for chemical analysis of the a-PZNZT films before HIP, and of crystallized films after HIP and polarization fatigue tests. The surface morphology and cross-sectional structure of PZNZT films were characterized by atomic force microscopy (AFM) and transmission electron microscopy (TEM). The relative permittivity $\left(\varepsilon_{\mathrm{r}}\right)$ and the dielectric loss $(\tan \delta)$ were measured using a precision LCR meter (4284A, Agilent). The polarization-electric field $(P-E)$ hysteresis loop and the polarization fatigue characteristic were measured using a ferroelectric film test system (RT66A, Radiant Technologies) and a function/arbitrary waveform generator (33120A, Hewlett-Packard). Polarization fatigue tests were performed using bipolar voltage pulses with amplitudes of $2.7-3.7 \mathrm{~V}$ at $500 \mathrm{kHz}$. The dc voltage offset calculated from measurement of the $P-E$ hysteresis loop was set so as to equalize the positive and negative fields applied to the sample.

\section{Results and Discussion}

\subsection{Crystal structure and orientation}

Figure 1 shows the XRD patterns and degree of $c$-axis orientation for PZNZT samples HIP-treated at $500^{\circ} \mathrm{C}$ for $1 \mathrm{~h}$ under gas pressures of $1.0-176.5 \mathrm{MPa}$, as well as those for the PZNZT sample before HIP for comparison. All the samples were confirmed to have a single-phase perovskite structure except for the untreated sample. Samples HIPtreated at $1.0 \mathrm{MPa}$ exhibited almost completely uniform (111) orientation. Two strong diffraction peaks at (001) and (101) appeared in samples HIP-treated at 1.3 MPa, and the orientation of samples treated at $1.8 \mathrm{MPa}$ changed to almost uniform (001), reaching $\alpha=0.95$. Although the preferred (001) orientation was retained in samples treated at $8.8-176.5 \mathrm{MPa}$, the crystallinity degraded with increasing HIP treatment pressure.

\subsection{AFM and TEM observations}

AFM images of (a) as-deposited PZNZT films at room temperature and HIP-treated films processed at (b) 1.8 and (c) 176.5 MPa are shown in Fig. 2. The surface structure of the amorphous film exhibits a characteristic striped pattern with relatively high root mean square roughness $\left(R_{\mathrm{ms}}\right)$, whereas the surface of the film treated at $1.8 \mathrm{MPa}$ has a high-density microstructure composed of small grains with an average grain size of around $80 \mathrm{~nm}$. It was found that the $R_{\mathrm{ms}}$ of all the HIP-treated films decreased to around half that of the sample before HIP and after RTA, demonstrating that HIP treatment provides significant improvements in surface flatness. Figure 3 shows the crosssectional high-resolution TEM images of the interfaces between the crystallized PZNZT layer and the PT seed layer, and between the PT seed layer and the bottom Pt electrode of PZNZT samples HIP-treated at (a) 1.8 and (b) 176.5 MPa. For the film treated at $1.8 \mathrm{MPa}$, the interface between the crystallized PZNZT layer and the PT seed layer is difficult to identify due to good adjustment of the two layers. This suggests that the crystallization of amorphous PZNZT film began at the PT seed layer. In contrast, the sample treated at 176.5 MPa exhibited several elongated amorphous areas oriented parallel to the substrate. These amorphous areas were approximately $8.0 \mathrm{~nm}$ wide and occurred in the

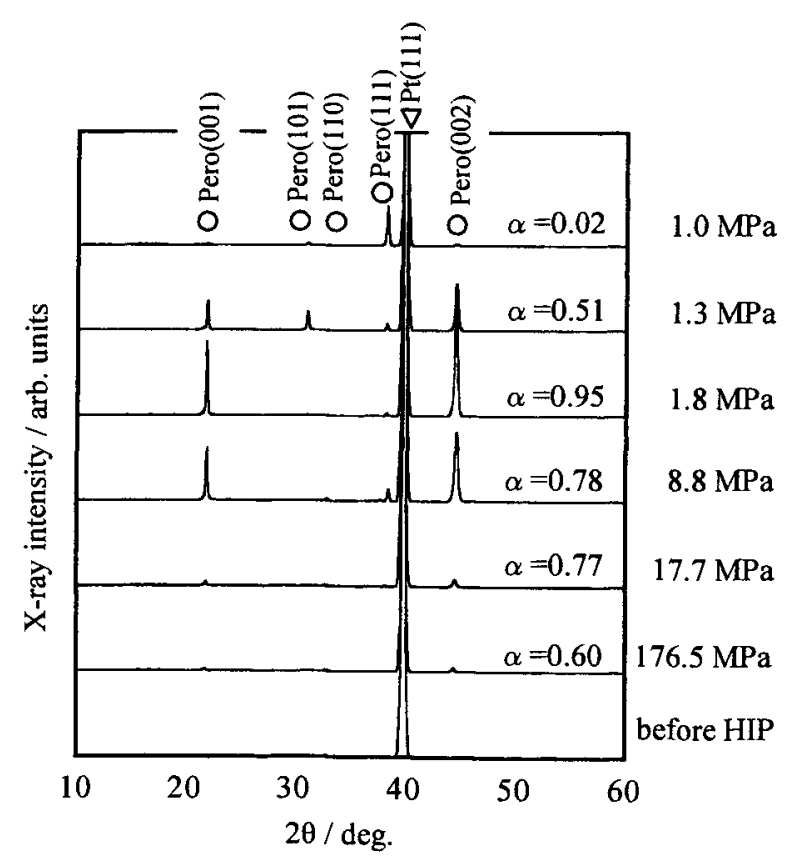

Fig.1 XRD patterns and degree of $c$-axis orientation for PZNZT samples HIP-treated at $500^{\circ} \mathrm{C}$ for $1 \mathrm{~h}$ under gas pressures of 1.0 to $176.5 \mathrm{MPa}$. 

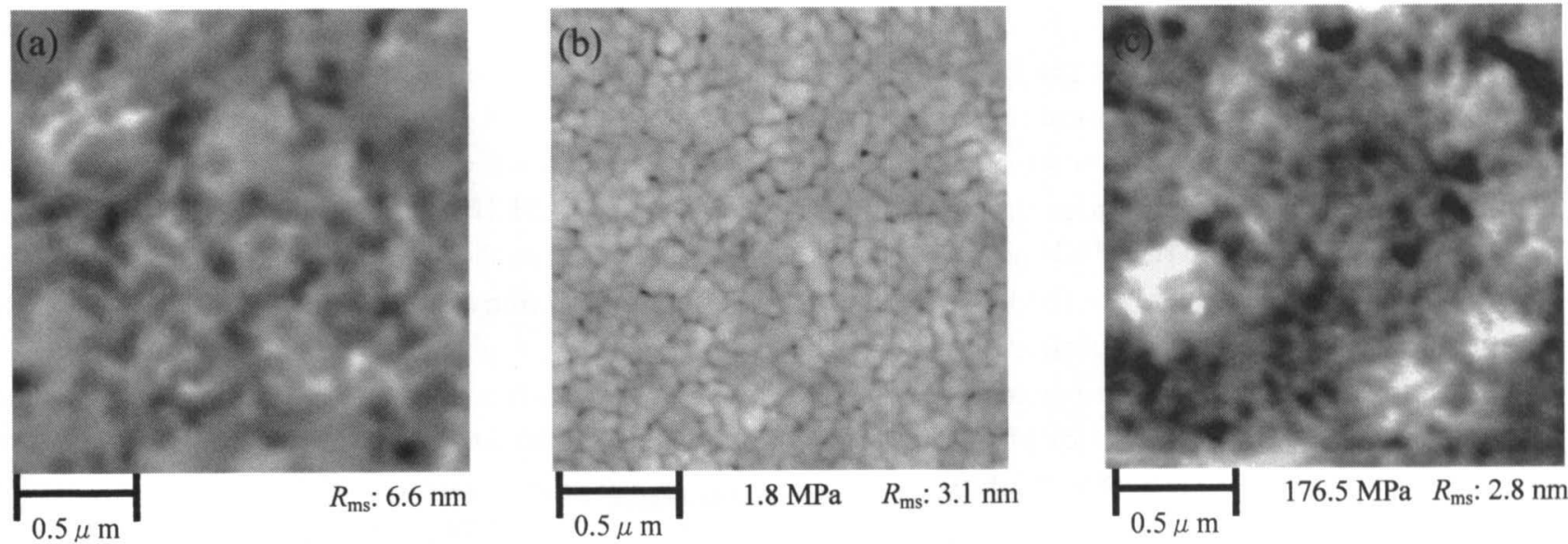

Fig.2 AFM images of (a) as-deposited PZNZT films at room temperature and HIP-treated films at $500^{\circ} \mathrm{C}$ for $1 \mathrm{~h}$ under (b) $1.8 \mathrm{MPa}$ and (c) $176.5 \mathrm{MPa}$.
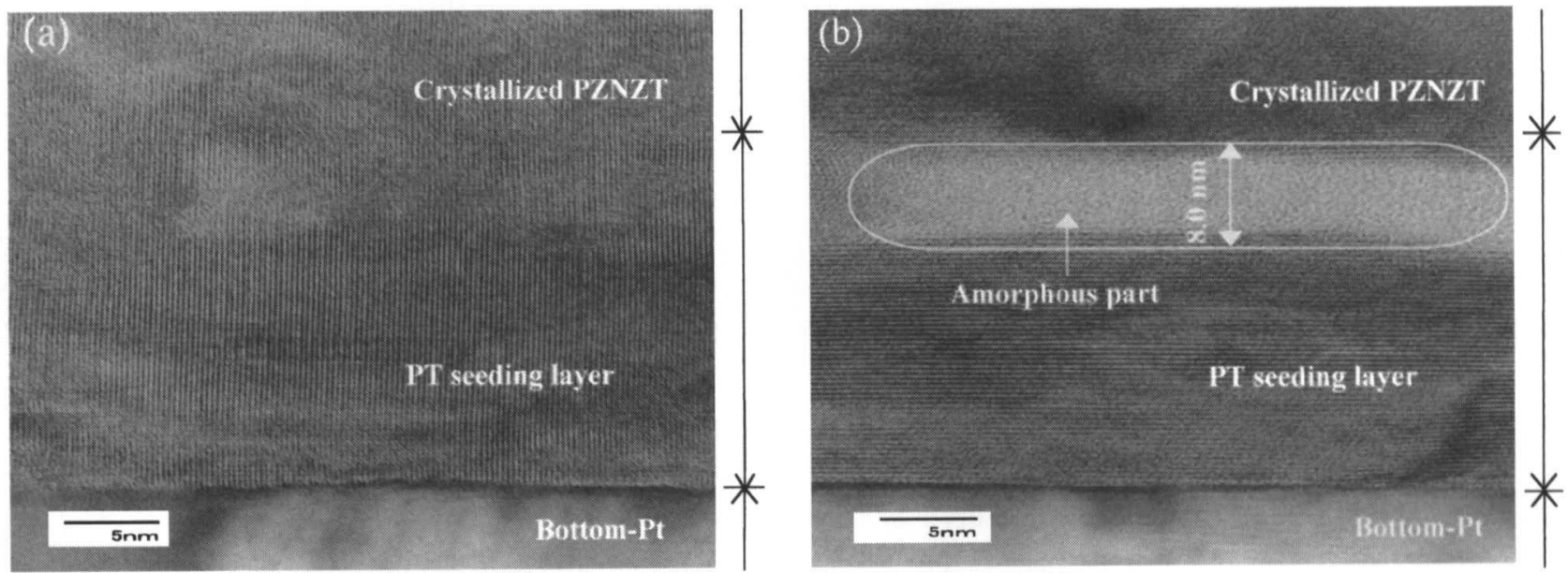

Fig.3 Cross-sectional high-resolution TEM images of the interfaces between the crystallized PZNZT layer and the PT seed layer, and between the PT seed layer and the bottom Pt electrode of PZNZT samples HIP-treated at $500^{\circ} \mathrm{C}$ for $1 \mathrm{~h}$ under (a) $1.8 \mathrm{MPa}$ and (b) $176.5 \mathrm{MPa}$.

vicinity of the interface between the crystallized PZNZT layer and the PT seed layer, suggesting that the amorphous part expanded gradually starting from the upper interface as the HIP pressure increased. As predicted from the previous XRD results, the degradation of crystallinity is considered to be due to an increase in the formation of this amorphous region in films with increasing HIP pressure. Therefore, to obtain crystallized films with few crystal defects, excellent surface flatness $\left(R_{\mathrm{ms}} \cong 3 \mathrm{~nm}\right)$ and minimal compositional fluctuation, HIP treatment at an appropriate pressure is significantly superior to conventional hightemperature sputtering.

\subsection{Dielectric properties}

The relative permittivity and dielectric loss of the PZNZT samples are shown in Fig. 4 as a function of HIP pressure. The relative permittivity tends to decrease linearly with

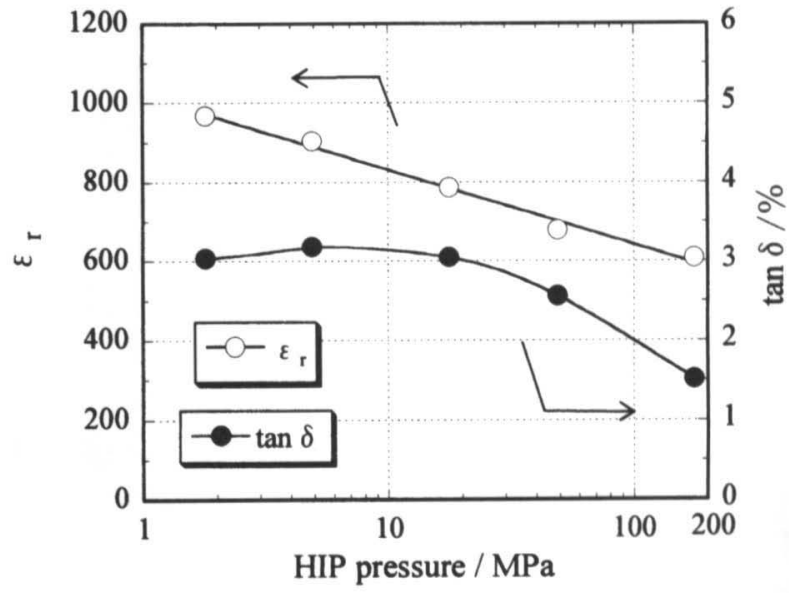

Fig.4 Relative permittivity and dielectric loss of PZNZT samples $\mathrm{HIP}$-treated at $500^{\circ} \mathrm{C}$ for $1 \mathrm{~h}$ under $1.8-176.5 \mathrm{MPa}$ as a function of HIP pressure. 


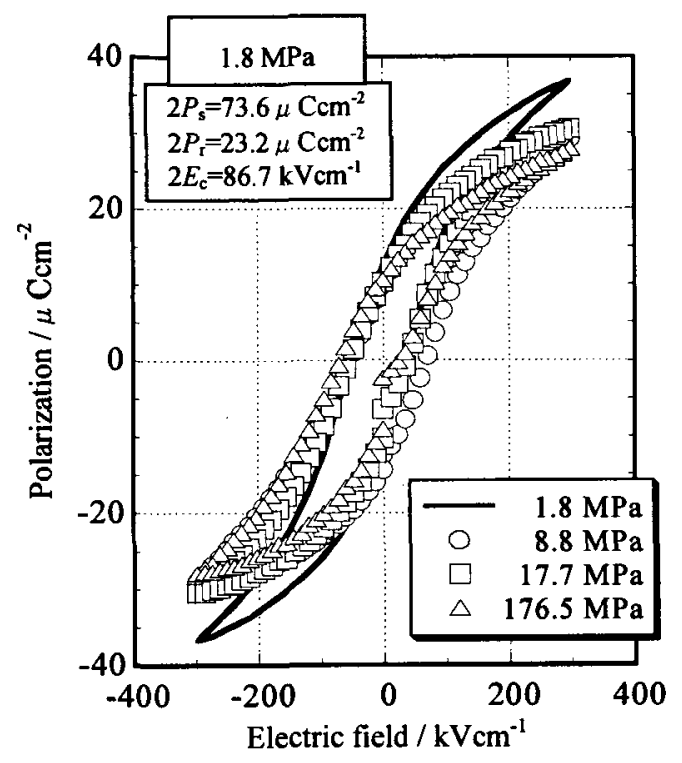

Fig.5 $P-E$ hysteresis loops of PZNZT samples crystallized at $500^{\circ} \mathrm{C}$ for $1 \mathrm{~h}$ under $1.8-176.5 \mathrm{MPa}$ by HIP.

increasing HIP pressure in the range 1.8-176.5 MPa, whereas the dielectric loss drops rapidly in the range 17.7-176.5 $\mathrm{MPa}$ after remaining almost constant $(\sim 3 \%)$ at lower pressures. By comparison with the XRD results and TEM observations, the decrease in relative permittivity can be explained by an increase in the extent of amorphous material with low $\varepsilon_{\mathrm{r}}$ as the HIP pressure increases. The nonlinear decrease in $\tan \delta$ in the range 17.7-176.5 MPa may be caused by densification of the microstructure.

3.4 Ferroelectric properties and polarization fatigue characteristics

Figure 5 shows the $P-E$ hysteresis loops of PZNZT samples crystallized by HIP. The $P-E$ characteristics for films treated at $1.8-176.5 \mathrm{MPa}$ correspond well with the XRD, TEM and $\varepsilon_{\mathrm{r}}$ results. The film treated at $1.8 \mathrm{MPa}$ exhibits a rectangular loop with a remanent polarization of $2 P_{\mathrm{r}}=23.2 \mu \mathrm{C} / \mathrm{cm}^{2}$ and a coercive field of $2 E_{\mathrm{c}}=86.7 \mathrm{kV} / \mathrm{cm}$. However, the $P-E$ loops of films treated at higher pressure become gradually flatter with increasing pressure. The polarization fatigue characteristics of typical PZNZT samples treated at $500^{\circ} \mathrm{C}$ for $1 \mathrm{~h}$ under $1.8-176.5 \mathrm{MPa}$ are shown in Fig. 6. The pulse-derived switchable polarization $\left(Q_{\text {sw }}\right)$ of the samples treated at 1.8 and $8.8 \mathrm{MPa}$ was initially high, decreasing gradually with increasing number of switching cycles, whereas samples treated at pressures greater than $17.7 \mathrm{MPa}$ exhibited relatively low initial $Q_{\mathrm{sw}}$ values that resisted an decrease even after up to $3 \times 10^{10}$ cycles. This fatigue-free behavior may be due to the formation of amorphous parts in the vicinity of the interface

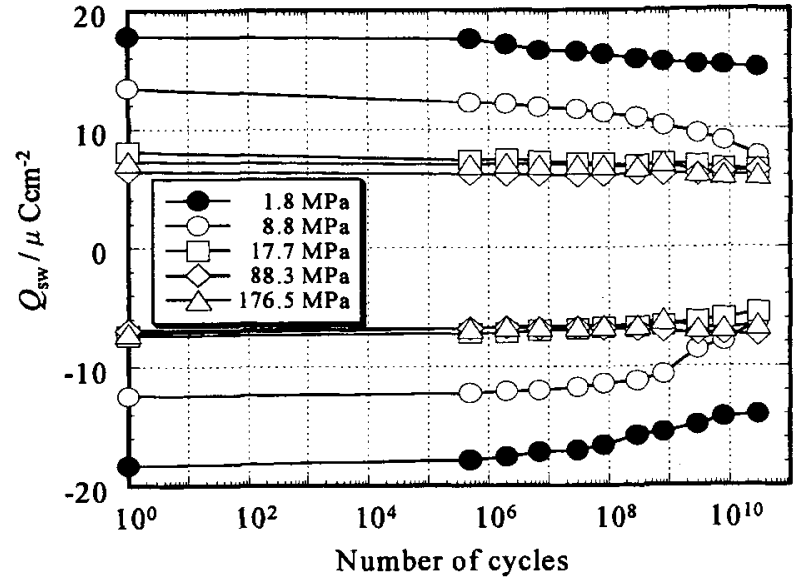

Fig.6 Polarization fatigue characteristics of typical Pt/PZNZT/PT/ Pt capacitors.

between the crystallized PZNZT layer and the PT seed layer, which would play an important role in restraining the diffusion of lead from the crystallized PZNZT film toward the PT seed layer. Moreover, the XRF measurements of the a-PZNZT films before HIP and the crystallized films after HIP, in conjunction with the results of polarization fatigue tests, indicate that chemical properties of films is largely constant, within the accuracy of measurement. This suggests that no appreciable compositional fluctuation occurs as a result of HIP treatment or polarization fatigue tests. Based on these results, the introduction of an appropriate fraction of amorphous structure in ferroelectric thin films can be expected to allow for the fabrication of fatigue-free capacitors that resist degredation even after $3 \times 10^{10}$ cycles in polarization fatigue tests using a simple Pt-ferroelectric-Pt structure with moderately high initial $Q_{\text {sw. }}$. This technology would be suitable for example in application to nonvolatile memory devices.

\section{Conclusions}

The results of this study can be summarized as follows.

(1) All the obtained films were confirmed to be of the singlephase perovskite structure in the present HIP pressure range of $1.0-176.5 \mathrm{MPa}$.

(2) The PZNZT films HIP-treated at $500^{\circ} \mathrm{C}$ for $1 \mathrm{~h}$ under 1.8 MPa showed almost complete (001) orientation and their degree of $c$-axis orientation, $\alpha$, reached up to 0.95 .

(3) The relative permittivity, $\varepsilon_{\mathrm{r}}$ decreased linearly in the range of 1.8-176.5 MPa due to increase in an amorphous part with low $\varepsilon_{\mathrm{r}}$ with increasing HIP pressure.

(4) The PZNZT films treated at $500^{\circ} \mathrm{C}$ for $1 \mathrm{~h}$ under a mixed gas pressure of $1.8 \mathrm{MPa}$ had symmetric and slim hysteresis 
loop shapes with a remanent polarization, $2 P_{\mathrm{r}}=23.2 \mu \mathrm{C} /$ $\mathrm{cm}^{2}$ and a coercive field, $2 E_{\mathrm{c}}=86.6 \mathrm{kV} / \mathrm{cm}$.

(5) All the samples treated at pressures greater than $17.7 \mathrm{MPa}$ completely showed fatigue-free characteristics even after up to $3 \times 10^{10}$ cycles.

\section{Acknowledgements}

This work was supported, in part, by a Research Grant from Himeji Institute of Technology and Taki Cultural Promotion Foundation which are gratefully acknowledged.

\section{References}

1) A.Kakimi, S.Okamura and T.Tsukamoto: " $\mathrm{O}_{2}$ Gas Pressure Effects on Surface Morphology in Ferroelectric $\mathrm{Bi}_{4} \mathrm{Ti}_{3} \mathrm{O}_{12}$ Thin Films Fabricated by Spin-Coating Pyrolysis", Jpn. J. Appl. Phys., 33(1994)L1707-L1709.

2) A.Kakimi, S.Okamura, S.Ando and T.Tsukamoto: "Effects of $\mathrm{O}_{2}$ Gas Pressure in Heat Treatment on Surface Morphology and Electric Properties of Ferroelectric $\mathrm{Bi}_{4} \mathrm{Ti}_{3} \mathrm{O}_{12}$ Thin Films with c-Axis Orientation", Jpn. J. Appl. Phys., 34(1995)5193-5197.

3) T.Kijima and H.Ishiwara: "Ultra-Thin Ferroelectric Films Modified by $\mathrm{Bi}_{2} \mathrm{SiO}_{5}$ ", Ferroelectrics, 271(2002) 289-295.
4) T.Kijima and H.Ishiwara: "Si-Substituted Ultrathin Ferroelectric Films", Jpn. J. Appl. Phys., 41 (2002) L716-L719.

5) T.Kijima and H.Ishiwara: "Si-Substituted Ultra-Thin Ferroelectric Films", Abstr. $8^{\text {th }}$ Conf. on Electronic Ceramics and Their Applications, (2002)8.

6) M.Kobune, S.Kojima, T.Yazawa and K.Honda: "Physical Properties of $0.24 \mathrm{~Pb}\left(\mathrm{Zn}_{1 / 3} \mathrm{Nb}_{2 / 3}\right) \mathrm{O}_{3}$. $0.384 \mathrm{PbZrO}_{3} \cdot 0.376 \mathrm{PbTiO}_{3}$ Thin Films Crystallized by Hot Isostatic Pressing", Jpn. J. Appl. Phys., 42(2003) 5963-5968.

7) M.Kobune, K.Muto and Y.Akiyama: "Piezoelectric and Ferroelectric Properties of $\mathrm{Pb}\left(\mathrm{Zr}_{1 / 3} \mathrm{Nb}_{2 / 3}\right) \mathrm{O}_{3}-\mathrm{PbTiO}_{3}$ $\mathrm{PbZrO}_{3}$ Ceramics", J. Ceram. Soc. Jpn., 110(2002)12-17.

8) M.Kobune, K.Muto, H.Takahashi, J.Qiu and J.Tani: "Effects of Microwave and Hot-Press Hybrid Sintering on Microstructure and Piezoelectric Properties of $0.24 \mathrm{~Pb}\left(\mathrm{Zn}_{1 / 3} \mathrm{Nb}_{2 / 3}\right) \mathrm{O}_{3} \cdot 0.384 \mathrm{PbZrO}_{3} \cdot 0.376 \mathrm{PbTiO}_{3}$ Ceramics", Jpn. J. Appl. Phys., 41 (2002)7089-7094.

9) M.Kobune, O.Matsuura, T.Matsuzaki, A.Mineshige, S.Fujii, H.Fujisawa, M.Shimizu and H.Niu: "Effects of $\mathrm{Pt} / \mathrm{SrRuO}_{3}$ Top Electrodes on Ferroelectric Properties of Epitaxial (Pb, La) $(\mathrm{Zr}, \mathrm{Ti}) \mathrm{O}_{3}$ Thin Films", Jpn. J. Appl. Phys., 39(2000)5451-5455. 